\title{
Ultrasonic biopsy needle based on the class IV flextensional configuration
}

\author{
Andrew Mathieson*, Andrew Feeney*, Andrew Tweedie ${ }^{\dagger}$, and Margaret Lucas* \\ ${ }^{*}$ School of Engineering, University of Glasgow, Glasgow, UK \\ ${ }^{\dagger}$ Thornton Tomasetti, Glasgow, UK \\ Email: andrew.mathieson@glasgow.ac.uk
}

\begin{abstract}
This study builds on previous research by the authors, where the design of a miniaturized class IV transducer configuration and adaption for including an end-effector for use in biopsy are investigated. Device design has focused on the generation of displacement uniformity across the output surface of the transducer. This has the aim of maximizing device reliability and minimizing nonlinear responses that could adversely affect the performance of devices incorporating endeffectors. The devices investigated in this study, designed using finite element analysis, have been experimentally characterized to identify their impedance and resonant characteristics.
\end{abstract}

\section{INTRODUCTION}

Flextensional transducers typically consist of piezoelectric or magnetostrictive transduction material sandwiched between two metal shells. Vibrational motion generated by the transduction material induces a combined flexural-extension motion of the shells which enables a high vibrational amplitude to be achieved at the output face of the device. First discussed by Hayes in 1936 [1], the variety of design concepts of flextensional devices has heralded seven classifications, I-VII. Although these were largely developed for use as underwater projectors [2], the miniaturized class V 'cymbal' configuration was initially developed as an actuator, demonstrating the capability to generate a sizable displacement across its output face for a low input energy and exhibiting a relatively high mechanical quality factor, $\mathrm{Q}_{\mathrm{m}}$ [3]. Cymbal transducers have more recently been employed in miniaturized power ultrasonic devices for orthopaedic procedures [4]. Driven via piezoceramic rings or discs and combined with a commercial resonant cutting blade, it was demonstrated that the cymbal device could cut hard tissue during bench top trials.

Despite the success of the cymbal configuration, it is anticipated that enhanced performance, per unit volume of piezoceramic material, could be achieved through the miniaturization of the class IV configuration. This study focuses on generating a uniform vibrational displacement across the endcap cavity. This is an important design consideration to ensure reliability and minimize nonlinear responses that could adversely affect the performance of devices incorporating end-effectors, such as cutting blades [5]. To demonstrate the feasibility of incorporating an end-effector to a miniaturized class IV device, a non-resonant needle designed for biopsy procedures has been fastened to the out put face via a thread joint.

\section{Flextensional DeVice}

Fig. 1 shows the flextensional device developed during this investigation. The transducer contains a PZ26 (Ferroperm Piezoceramics) bar with the dimensions $50 \mathrm{~mm} \times 10 \mathrm{~mm} \times$ $5 \mathrm{~mm}$, and a backmass and endcap which are both manufactured from CZ112 naval brass. The devices are tuned to resonant at the length mode of the piezoceramic bar.

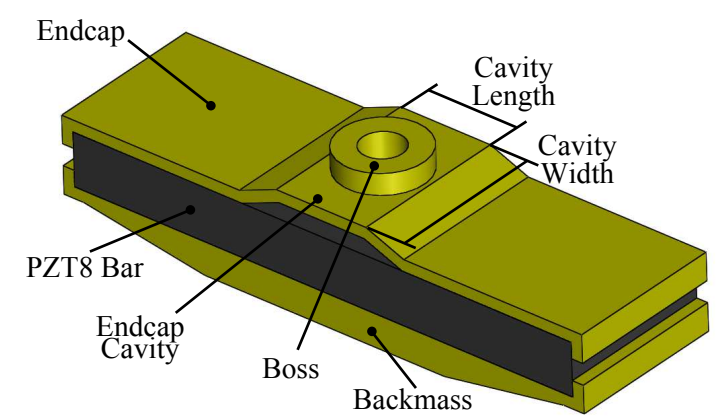

Fig. 1. Class IV flextensional transducer developed during this study

\section{A. Endcap design considerations}

Preliminary design considerations focused on the generation of an uniform amplitude displacement across the endcap output face. A non-uniform vibrational amplitude, across the output surface of a power ultrasonic device fastened to a slender tool, can induce nonlinear vibrational responses, such as modal coupling, which can lead to device instability, premature failure and sub-optimal device performance [5].

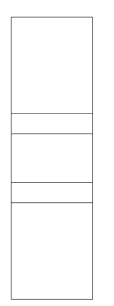

(a)

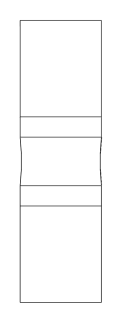

(b)

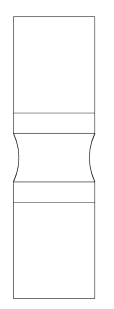

(c)

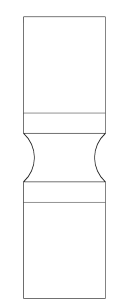

(d)

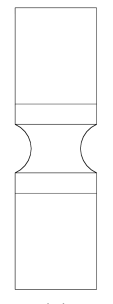

(e)

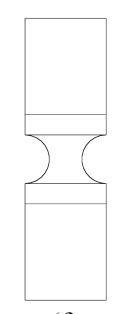

(f)
Fig. 2. Top view of endcaps with arc radius; (a) $0.00 \mathrm{~mm}$, (b) $20.50 \mathrm{~mm}$, (c) $8.72 \mathrm{~mm}$, (d) $6.10 \mathrm{~mm}$, (e) $5.10 \mathrm{~mm}$, and (f) $4.60 \mathrm{~mm}$ 
The vibrational characteristics of the flextensional device were modelled using the finite element analysis software, Abaqus (Dassault Systèmes). A rectangular endcap cavity profile was considered before incorporating convex geometry, which resembles a 'bow-tie', with decreasing radius. The normalized vibrational response through the center of the width and length of the endcaps whilst at their tuned mode of vibration are presented in Fig. 3. It can be observed that with a decrease in radius, vibrational uniformity can be improved across the width of the endcap. Although an increase in the maximum amplitude of vibration is predicted along the length of the cavity, no significant improvement in vibrational uniformity is achieved.

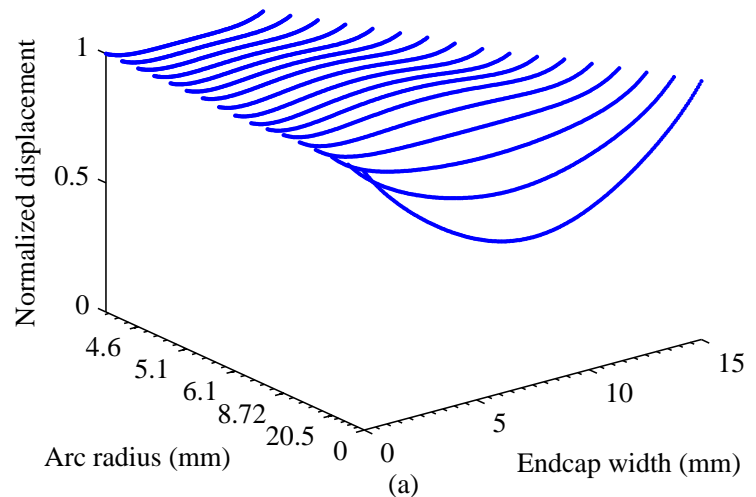

(a)

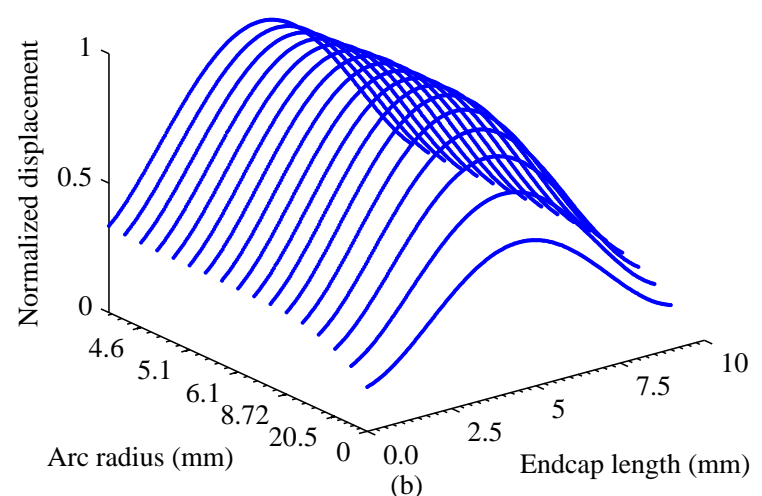

Fig. 3. Normalised displacement across (a) Cavity width, and (b) Cavity length

\section{B. Addition of needle}

To enable the attachment of an end-effector, such as a needle, via a threaded joint, a boss has been incorporated on to the output surface of the endcap as shown in Fig. 1. The normalised vibrational amplitude across the output surface of the boss is presented in Fig. 3. By comparing Fig. 3 and Fig. 4 , it can be observed that vibrational uniformity across both directions is improved with the addition of the boss, and it is also evident that the convex geometry with the smallest radius is predicted to exhibit the largest vibrational gain.

The needle which has been designed to be incorporated with the flextensional device has been based on a gauge 8 standard

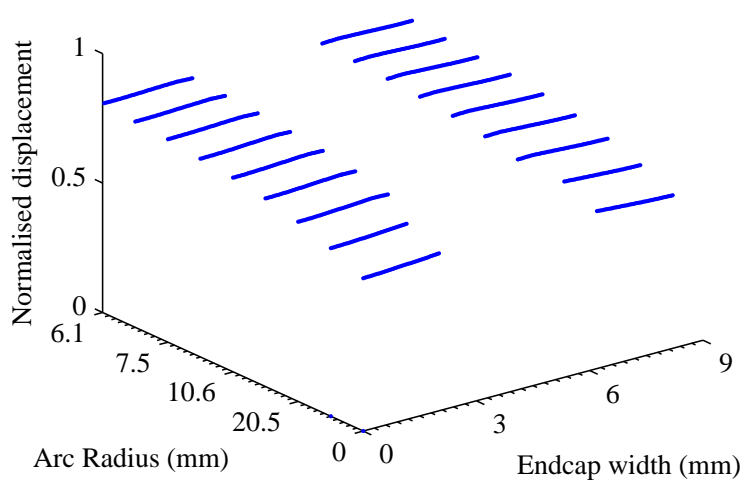

(a)

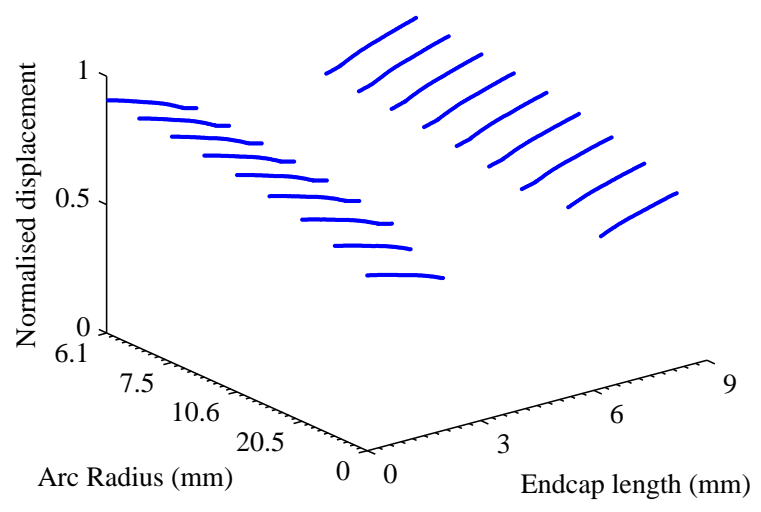

(b)

Fig. 4. Normalised displacement across boss (a) Cavity width, and (b) Cavity length

needle design and is presently used in clinical procedures. It has an OD: $4.20 \mathrm{~mm}$, ID: $3.10 \mathrm{~mm}$, and length: 44.90 $\mathrm{mm}$, and is manufactured from Ti6Al4V. It has been designed to act has a rigid body at the operational frequency of the flextensional transducer. The initial needle concept consisted of a tube with a uniform thickness, which fastens into an M5 threaded hole located in the boss. However, it was observed from FEA predictions that breathing motion was induced in the needle wall by the motion generated by the endcap cavity, and is shown in Fig. 5. The presence of this motion could could induce undesirable vibrational behaviors, such as modal coupling, or even inflict damage to extracted biopsy tissue.

The normalized $\mathrm{x}$-direction vibrational component of the breathing motion, which is that perpendicular to the longitudinal needle axis, of the needle was extracted along the measurement path indicated in Fig 5. Fig. It can be seen from Fig. 6(a) that the largest motion corresponds to the location where the base of the needle is fastens to the endcap. It is also clear that a convex endcap geometry induces a larger displacement in the $\mathrm{x}$-direction than a rectangular endcap geometry. As seen in Fig. 6(b), the displacement induced by the breathing motion was significantly reduced through employing an endcap with a rectangular cavity profile and by incorporating a conical section into the base. 


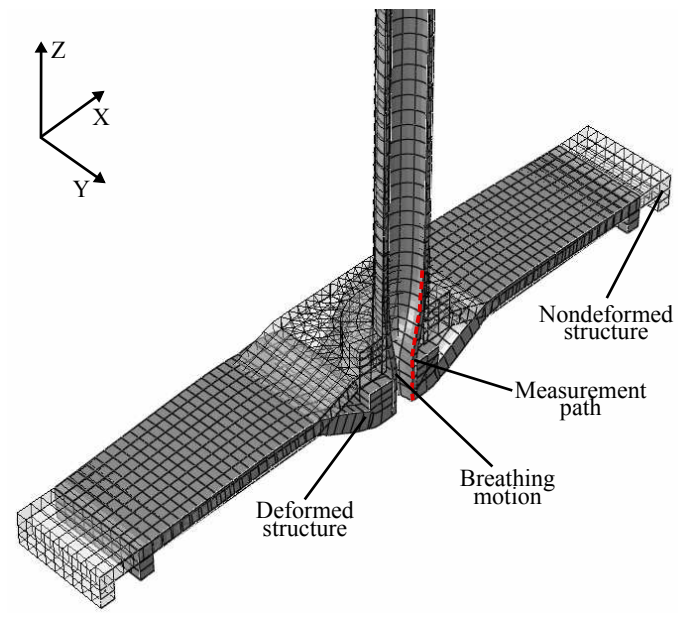

Fig. 5. Deformed and undeformed section of endcap and needle
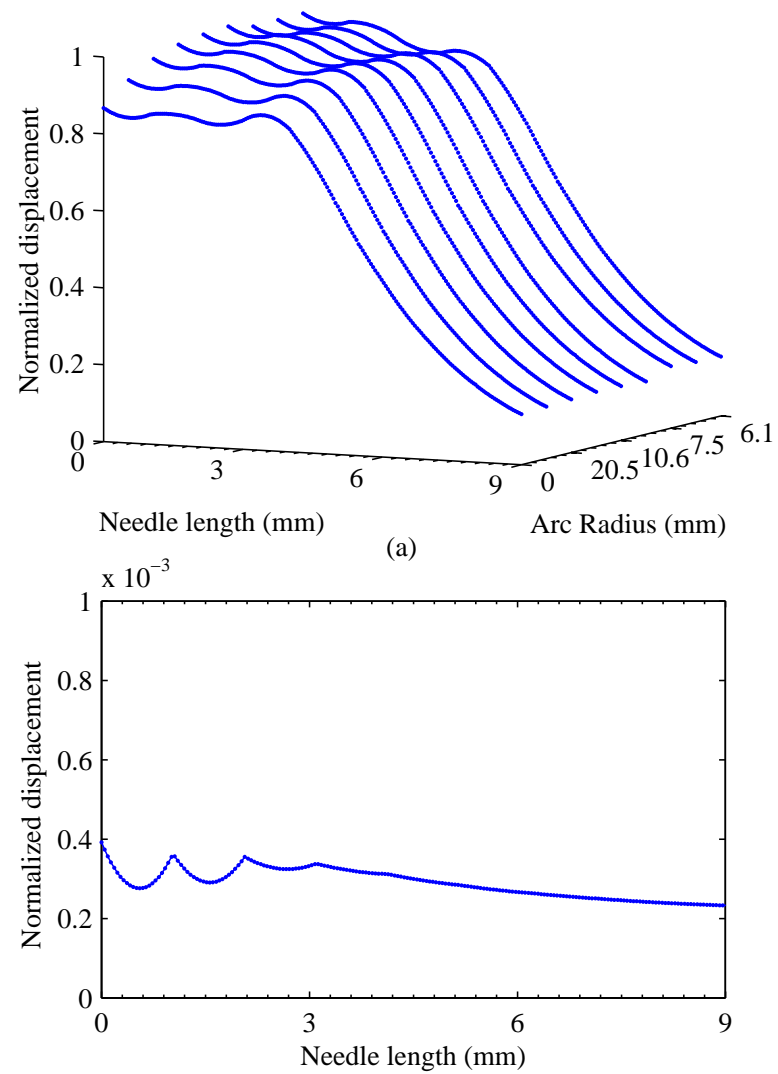

(b)

Fig. 6. Normalised displacement. Measured in X-dir ${ }^{\mathrm{n}}$ from needle base for (a) Endcap geometry with a reducing arc radius, and (b) Device with a rectangular cavity profile and needle incorporating a conical section

\section{DEVICE FABRICATION}

Two geometrically identical flextensional devices were fabricated, with one shown in Fig. 7. Device 1 was assembled with insulating epoxy resin (Eccobond 45LV High Strength, Ellsworth Adhesives Ltd.) acting as the mechanical coupling agent between the PZ26 bar and the endcap and backmass. Device 2 was assembled using the same epoxy resin as the mechanical coupling between the backmass and PZ26 bar, while the front mass was coupled to the PZ26 bar via an interference fit.

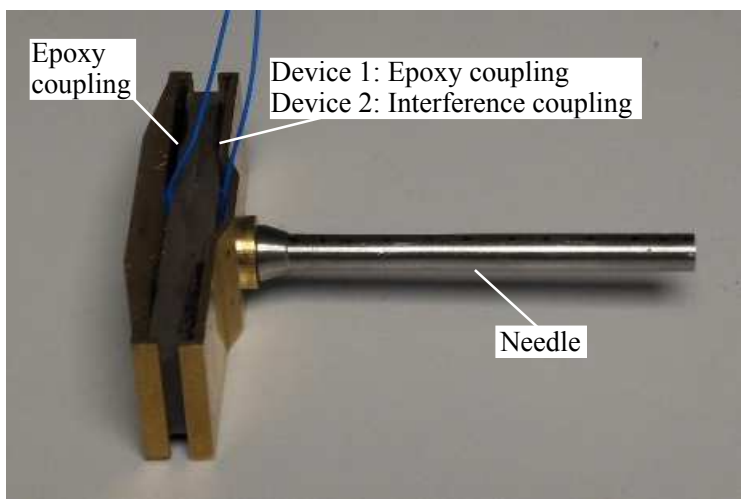

Fig. 7. Flextensional - needle assembly

\section{EXPERIMENTAL CHARACTERIZATION}

\section{A. Impedance analysis}

Fig. 8 presents impedance and phase plots from the fabricated transducers which were collected using a 4194A Impedance/Gain-Phase Analyzer (Hewlett Packard). It can be seen from Fig. 8 that the response of Device 2 is greater than Device 1, highlighting the dampening effect of the epoxy resin. It can also be seen from Fig. 8 that Device 1 exhibits a response close to $20 \mathrm{kHz}$, while Device 2 does not. This is likely to stem from an uneven distribution of the epoxy between the endcap and PZ26 bar, causing localised variances of bond strength and stiffness. The considerably lower impedance of Device 2 at resonance was found to be misleading. Further characterisation (through experimental modal analysis) of Device 2 demonstrated that the endcap was not successfully coupling with the PZ26 bar and that the endcap did not resonate.

\section{B. Experimental modal analysis}

Experimental modal analysis (EMA) was performed to identify the resonant frequency and mode shape of the tuned mode of vibration of the devices. The needle assembly was excited via a low power random signal (generated by a Data Physics Quattro and amplified via an QSC RMX 4050HD power amplifier), while the velocity responses were measured across a grid of points located on the surfaces of the endcap, backmass and needle using a 3-D laser Doppler vibrometer (Polytec, CLV-3D). The frequency response functions were acquired and recorded using data acquisition hardware and software (Data Physics Quattro and Signal Calc ACE software) with a resolution of $1.6 \mathrm{~Hz}$ before the resonant frequency and mode shape of the tuned mode of vibration were identified, extracted and visualised using ME'Scope software (Vibrant Technology Inc.).

The resonant frequency of the tuned mode of vibration was predicted using FEA at $35294 \mathrm{~Hz}$, while the measured 


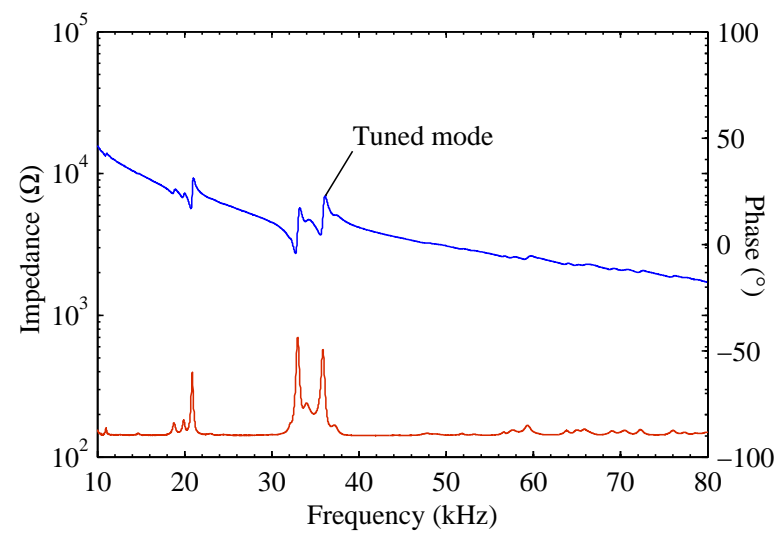

(a)

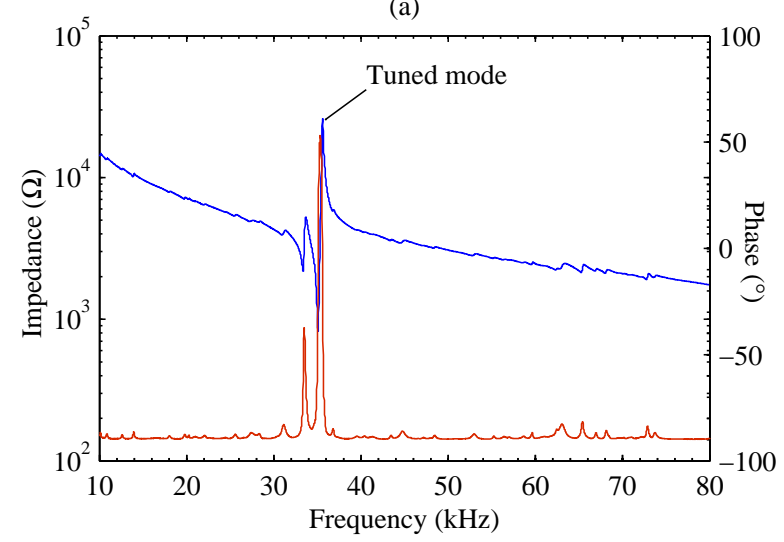

(b)

Fig. 8. Impedance and phase plots from (a) Device 1, and (b) Device 2

resonant frequency was found to be $35129 \mathrm{~Hz}$ using EMA. The percentage difference between these frequencies of $0.5 \%$ was lower than expected. Previous research has indicated that the layer of epoxy resin can be difficult to model and its presence in the fabricated device can cause inaccuracies in FEA models [6]. It is likely that the accuracy of the predicted frequency of the tuned mode of vibration has been enhanced by the incorporation of the needle end-effector. Although the incorporation of an end-effector do not significantly influence the resonant frequency of the flextensional device [4], its addition has increased the size of the device, which could have reduced the influence that the epoxy resin has on the vibrational response.

Fig. 9 presents the mode shape of the tuned mode of vibration which were predicted using FEA and measured by EMA. Vibrational displacement is represented using a contour plot, where blue shades represent locations of low vibrational amplitude and red shades represent locations of high vibrational amplitude. The mode shape predicted by FEA has been found to closely match the mode shape identified through EMA. The node located on the needle is in a similar position in the predicted and measured mode shapes, while the edge of the endcap cavity can be seen to curl at the same location. However, differences between the predicted and measured mode shapes can also be observed. The asymmetry in the vibrational response of the endcap can be attributed to

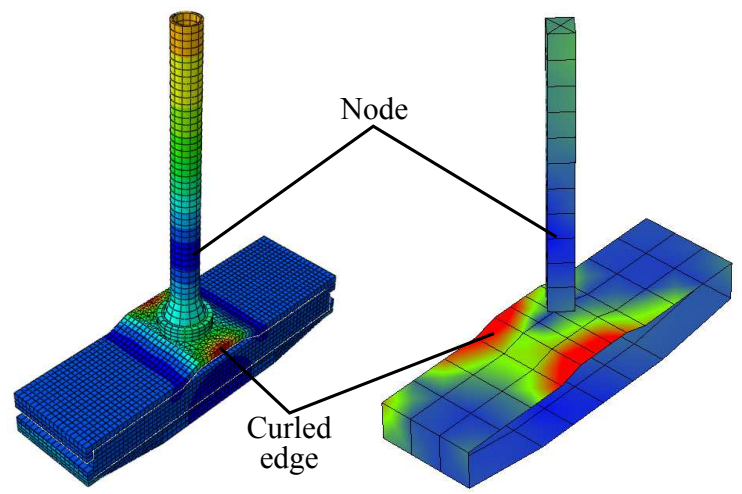

(a)

(b)

Fig. 9. Mode shape of tuned mode of vibration (a) predicted through FEA, and (b) measured from EMA

the uneven deposition of the epoxy resin [6].

\section{CONCLUSION}

This study reports on a miniaturized class IV flextensional transducer with an endcap cavity that resonates with a uniform vibrational displacement, and which has been modified to incorporate a needle end-effector. It was found that the modal parameters predicted by FEA correlated with those measured using EMA much closer than expected. Despite this, device fabrication methods have been highlighted as a significant limiting factor. Although epoxy resin provides a relatively strong mechanical coupling between the endcap and the PZ26 bar, it confers a significant amount of damping to the vibrational response, and its uneven distribution induces asymmetry in the vibrational response of the device. Meanwhile, poor mechanical coupling between the endcap and the PZ26 bar, resulting from the interference fit, in Device 2 indicates that a different design is required to create a successful interference coupling between the endcap and piezoceramic element which would be suitable for a power ultrasonic needle device.

\section{ACKNOWLEDGMENT}

This project is funded by the Engineering and Physical Science Research Council (EPSRC), grant number $\mathrm{EP} / \mathrm{K} 020013 / 1$.

\section{REFERENCES}

[1] H.C. Hayes, "Sound generating and directing apparatus," U.S. Patent 2 064 911, Dec. 22, 1936.

[2] K. Rolt, "History of the flextensional electroacoustic transducer," The Journal of the Acoustical Society of America, vol. 87, pp. 1340-1349, 1989.

[3] J. Zhang, A-C. Hladky-Hennion, W.J. Hughes, R.E. Newnham, "'Modeling and Underwater Characterization of Cymbal Transducers and Arrays," IEEE Trans. Ultrason. Ferroelectr. Freq. Control, vol. 48, pp.560-568, 2001.

[4] F. Bejarano, A. Feeney, M. Lucas, "A cymbal transducer for power ultrasonics applications," Sensor Actuat. A-Phys., vol. 210, pp. 182-189, 2014.

[5] A. Cardoni, M. Lucas, "Enhanced vibration performance of ultrasonic block horns", Ultrasonics, vol. 40, pp. 365-369, 2002.

[6] P. Ochoa, J.L. Pons, M. Villegas, and J.F. Fernandez. "Effect of bonding layer on the electromechanical response of the cymbal metal-ceramic piezocomposite," J. Eur. Ceram. Soc., vol. 27, pp. 1143-1149, 2007. 